\title{
Speed and surge control for a low order centrifugal compressor model
}

\author{
Jan Tommy Gravdahl and Olav Egeland \\ Department of Engineering Cybernetics \\ Norwegian University of Science and Technology \\ O.S. Bragstads plass 8, N-7034 Trondheim, Norway \\ Email: \{Tommy.Gravdahl,Olav.Egeland\}@itk.ntnu.no
}

\begin{abstract}
Previous work on stabilization of compressor surge is extended to include control of the angular velocity of the compressor. First a low order centrifugal compressor model is presented where the states are mass flow, pressure rise and rotational speed of the spool. Energy transfer considerations are used to develop a compressor characteristic. In order to stabilize equilibriums to the left of the surge line, a close coupled valve is used in series with the compressor. Controllers for the valve pressure drop and spool speed are derived. Semi-global exponential stability is proved using a Lyapunov argument.
\end{abstract}

\section{Introduction}

Compressor surge is an axisymmetric oscillation of the mass flow and pressure rise. Modeling and control of these oscillations is of considerable interest since surge limits the useful range of mass flows where the compressor operate stably. Large amplitude surge can also damage the compressor. A low order lumped parameter model capable of simulating these oscillations was first introduced in [10], where a model for axial compressor mass flow and pressure rise was presented. In [13] it was shown that the model of [10] is also applicable to centrifugal (radial) compressors.

Over the last decade many papers covering the area of surge control have been published. A recent review can be found in [2]. Of many possible actuation schemes, closed coupled valve $(\mathrm{CCV})$ control is considered one of the most promising $[4,11,12,14,16]$. Experimental results of CCV control is reported in [4] and [12]. The cause of surge in a compression system is that the throttle line crosses the compressor characteristic in an area of positive compressor characteristic slope. A close coupled valve is placed immediately downstream of the compressor (hence close coupled), and active control of the valve pressure drop is utilized to make the slope of the equivalent compressor (compressor in series with the valve) negative and thereby stabilizing the system.

This approach was used in [12], [14] and [16] for surge control of the model of [10]. Linear stability analysis was used in designing control laws resulting in local stability results. In [15] pressure disturbances were included in the analysis, and a nonlinear CCV control law was designed for the model of [10] using Lyapunovs method. By applying backstepping [8] developed nonlinear surge controllers for the same model, but included disturbances both in mass flow and pressure. Global stability results was presented. In [9] certain passivity properties of the model was utilized in designing a CCV control law.

One drawback of $\mathrm{CCV}$ control is that the valve introduces a pressure drop in the compression system as discussed in [15]. When using the valve as a steady-state device, such as in [4], this loss may become unacceptably large. However, as pointed out in [14] and [15], a time varying valve will introduce considerably less pressure drop than a valve with constant pressure drop.

Since compressor are variable speed machines, it is of interest to investigate the influence of speed transients on the surge dynamics. Models describing this interaction was developed in [5] for axial compressors and in [7] for centrifugal compressors. As surge can occur during acceleration of the compressor speed, it is of major concern to develop controllers that simultaneously can control both surge and compressor speed.

In this paper we present a surge controller for a variable speed centrifugal compressor. The speed is controlled with a PIcontrol law. Inspired by [6] and [17], we make a departure from the 3rd order polynomial approximation of the compressor characteristic commonly used in the literature. Fluid friction and incidence losses in the compressor stage are modeled, and a variable speed compressor characteristic is developed based on this. Semi-global exponential stability results for the proposed controllers are given using Lyapunovs method. The results are confirmed through simulations.

\section{Preliminaries}

We are considering a compression system consisting of a centrifugal compressor, close coupled valve, compressor duct, plenum volume and a throttle. The throttle can be regarded as a simplified model of a turbine. The system is showed in figure 2.a. The model to be used for controller design is in the form

$$
\begin{aligned}
\dot{p}_{p} & =\frac{a_{01}^{2}}{V_{p}}\left(m-m_{t}\right) \\
\frac{L_{c}}{A_{1}} \dot{m} & =p_{2}-p_{p} \\
J \dot{\omega} & =\tau_{t}-\tau_{c}
\end{aligned}
$$

where $m$ is the compressor mass flow, $p_{2}$ is the pressure downstream of the compressor, $a_{01}$ is the inlet stagnation sonic velocity, $L_{c}$ is the length of compressor and duct, $A_{1}$ is the area of the impeller eye (used as reference area), $J$ is the spool moment of inertia, $\tau_{t}$ is the drive torque and $\tau_{c}$ is the compressor torque. The two first equations of (1) are equivalent to the model of [10].

The angular speed of the compressor $\omega$ is included as a state in addition to mass flow and pressure rise which are the states in Greitzers surge model. The equation for $\dot{p}_{p}$ follows from the mass balance in the plenum, assuming the plenum process isentropic, and the equation for $\dot{m}$ follows from the impulse balance in the duct. In the following, the model (1) will be developed in detail. In particular, expressions must be found for the terms $p_{2}$ and $\tau_{c}$. It will also be shown that an expression for the compressor characteristic results from this derivation.

Incoming gas (air) enters the impeller eye (the inducer) of the compressor with velocity $C_{1}$, see figure $2 . \mathrm{b}$. The mass flow $m$ and $C_{1}$ is given by

$$
C_{1}=\frac{1}{\rho_{01} A_{1}} m
$$

where $\rho_{01}$ is the assumed constant stagnation inlet density. The tangential velocity $U_{1}$ of the inducer is calculated as

$$
U_{1}=\frac{D_{1}}{2} \omega=D_{1} \pi N,
$$




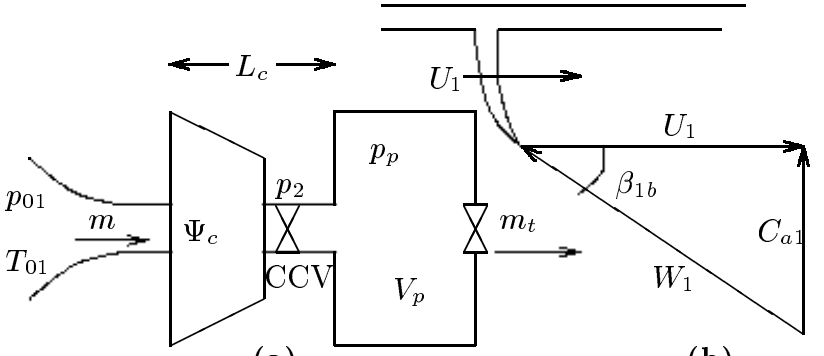

(a)

(b)

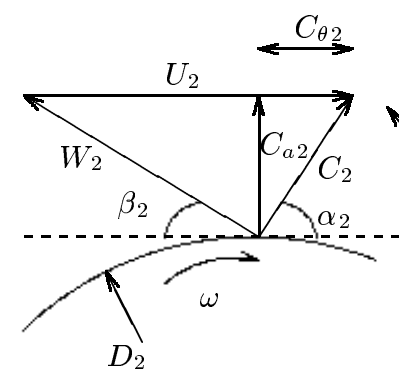

(c)

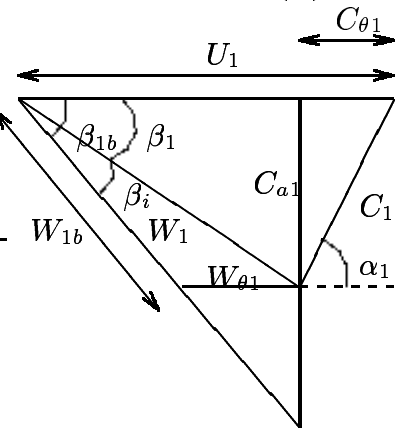

(d)

Figure 1: (a)Compression system, (b)Velocity triangle at inducer, (b)Velocity triangle at impeller tip and (d)Incidence angles

where $\omega$ is the angular velocity of the impeller and $N$ is the number of revolutions per second. The average diameter $D_{1}$ is defined according to

$$
D_{1}^{2}=\frac{1}{2}\left(D_{t 1}^{2}+D_{h_{1}}^{2}\right)
$$

where $D_{t 1}$ and $D_{h_{1}}$ are the diameters at inducer tip and hub casing respectively. The circle with diameter $D_{1}$ and area $A_{1}$ divides the inducer in two annuli of equal area. The compressed gas leaves the impeller at the impeller tip with velocity $C_{2}$ as showed in figure 2.c. The diameter at the impeller tip is $D_{2}$ and the tangential tip velocity is $U_{2}$.

\section{Energy transfer and compressor torque}

\subsection{Ideal case}

Eulers turbomachinery equations state that applied torque equals the change in angular momentum of the fluid

$$
\tau_{c}=m\left(r_{2} C_{\theta 2}-r_{1} C_{\theta 1}\right)
$$

where $\tau_{c}$ is the compressor torque, $r_{1}=\frac{D_{1}}{2}, r_{2}=\frac{D_{2}}{2}$ and $C_{\theta 2}$ is the tangential component of the gas velocity $C_{2}$. Power delivered to the fluid is

$$
\begin{aligned}
\dot{W}_{c} & =\omega \tau_{c}=\omega m\left(r_{2} C_{\theta 2}-r_{1} C_{\theta 1}\right) \\
& =m\left(U_{2} C_{\theta 2}-U_{1} C_{\theta 1}\right)=m \Delta h_{0 c, \text { ideal }}
\end{aligned}
$$

where $\Delta h_{0 c, \text { ideal }}$ is the specific enthalpy delivered to the fluid without taking account for losses. Equation (6) is known as Eulers pump equation. For simplicity the following two assumptions are made. A radially vaned (no backsweep) impeller is considered with $\beta_{2 b}=90^{\circ}$, and there is no pre-whirl, that is $\alpha_{1}=90^{\circ} \Rightarrow C_{\theta 1}=0$. The slip factor is defined as

$$
\sigma \triangleq \frac{C_{\theta 2}}{U_{2}} \approx 1-\frac{2}{i}
$$

The approximation is known as Stanitz' formula where $i$ is the number of compressor blades. From (6), and using (7), we have that the ideal specific enthalpy delivered to the fluid is

$$
\Delta h_{0 c, \text { ideal }}=\frac{\dot{W}_{c, \text { ideal }}}{m}=\sigma U_{2}^{2}
$$

and that the compressor torque is

$$
\tau_{c}=m r_{2} C_{\theta 2}=m r_{2} \sigma U_{2} .
$$

Notice that $\Delta h_{0 c, \text { ideal }}$ is independent of mass flow $m$, and ideally we would have the same energy transfer for all mass flows. However, due to various losses, the energy transfer is not constant, and we now include this in the analysis. According to [17], the two major losses, expressed as specific enthalpies, are: 1) Incidence losses, $\Delta h_{i}$ and 2) Fluid friction losses, $\Delta h_{f}$ Other losses such as inlet casing losses, disc friction losses, leakage losses and collector losses do occur, but these will not be considered here. Moreover, the incidence losses and fluid friction losses play an important role in determining the region of stable operation for the compressor.

\subsection{Incidence losses}

The velocity of the incoming gas relative to the inducer is denoted $W_{1}$. In off-design operation there will be a mismatch between the fixed blade angle $\beta_{1 b}$ and the direction of the gas stream $\beta_{1}=\beta_{1}\left(U_{1}, C_{1}\right)$, as showed in figure 2.d. The angle of incidence is defined by

$$
\beta_{i} \triangleq \beta_{1 b}-\beta_{1}
$$

As the gas hits the inducer, its velocity instantaneously changes its direction to comply with the blade inlet angle $\beta_{1 b}$. The direction is changed from $\beta_{1}$ to $\beta_{1 b}$, and the energy associated with the tangential component $W_{\theta 1}$ of the velocity is lost. That is, the incidence loss can be expressed as

$$
\Delta h_{i}=\frac{W_{\theta 1}^{2}}{2}
$$

From figure 2.d it is easily seen that

$$
\cos \beta_{1}=\frac{U_{1}-C_{\theta 1}}{W_{1}} \text { and } \sin \beta_{1}=\frac{C_{a 1}}{W_{1}} .
$$

Furthermore

$$
W_{\theta 1}=\frac{\sin \left(\beta_{1 b}-\beta_{1}\right)}{\sin \beta_{1 b}} W_{1}=\left(\cos \beta_{1}-\cot \beta_{1 b} \sin \beta_{1}\right) W_{1} .
$$

Inserting (12) in (13) gives

$$
W_{\theta 1}=U_{1}-C_{\theta 1}-\cot \beta_{1 b} C_{a 1} .
$$

and the incidence loss (11) can be written

$$
\Delta h_{i}=\frac{1}{2}\left(U_{1}-C_{\theta 1}-\cot \beta_{1 b} C_{a 1}\right)^{2}=\frac{1}{2}\left(U_{1}-\frac{\cot \beta_{1 b} m}{\rho_{01} A_{1}}\right)^{2}
$$

where the second equality if found using (2). Similar results are presented in chap. 5 in [6].

\subsection{Frictional losses}

According to [6] loss due to friction can be calculated as

$$
\Delta h_{f}=C_{h} \frac{l}{D} \frac{W_{1 b}^{2}}{2},
$$

where $C_{h}$ is the surface friction loss coefficient , $l$ is the mean channel length and $D$ is the mean hydraulic channel diameter. This friction loss is actually calculated for constant area pipes of circular cross section. The friction loss coefficient $C_{h}$ is defined as [17]

$$
C_{h}=4 f
$$

where the friction factor $f$ depends on the Reynolds number Many different formulas for the friction factor have been published, see e.g. [6] or [18]. Here we will use Blasius' formula

$$
f=0.3164(R e)^{-0.25}
$$

which was found empirically for turbulent flow in smooth pipes with Reynolds number Re below 100.000 [18]. The mean hydraulic channel diameter $D$ is defined as

$$
D=\frac{4 A}{a}
$$


where the cross section area $A$ and perimeter $a$ are mean values for the passage. The mean hydraulic diameter $D$ corresponds to a circle with area $A$ and perimeter $a$. Although the passages between the blades in the compressor are neither circular nor of constant area, [1] reports of good agreement between theory and measurement using (18).

Using figure 2.d, it is seen that

$$
\frac{W_{1 b}}{\sin \beta_{1}}=\frac{W_{1}}{\sin \beta_{1 b}}
$$

and using $\sin \beta_{1}=\frac{C_{a 1}}{W_{1}}$ we get

$$
W_{1 b}=\frac{C_{1}}{\sin \beta_{1 b}} .
$$

Inserting (2) and (21) in (16) gives

$$
\Delta h_{f}=\frac{C_{h} l}{2 D \rho_{1}^{2} A_{1}^{2} \sin ^{2} \beta_{1 b}} m^{2}=k_{f} m^{2} .
$$

As can be seen the friction losses are quadratic in mass flow and independent of wheel speed $U$. Equation (22) represents the loss due to friction of a mass flow $m$ through a pipe of hydraulic diameter $D$.

\section{Energy transfer and pressure rise}

Including the losses, the total specific energy transfer can be calculated by subtracting (22) and (15) from (8):

$$
\Delta h_{0 c}\left(U_{1}, m\right)=\Delta h_{0 c, i d e a l}-\Delta h_{f}-\Delta h_{i} .
$$

$\Delta h_{0 c}$ is a 2 nd degree polynomial in $m$, and as opposed to the ideal case, we see that energy transfer to the fluid is varying with mass flow $m$. This is showed in figure 2 .

To find an expression for the pressure rise we now need a relation between pressure rise and energy transfer. The pressure rise is modeled as

$$
p_{2}=\left(1+\frac{\Delta h_{0 c}\left(U_{1}, m\right)}{T_{01} c_{p}}\right)^{\frac{\gamma \eta_{p o l}}{\gamma-1}} p_{01}=\Psi_{c}\left(U_{1}, m\right) p_{01},
$$

where the losses have been taken into account, and $\Psi_{c}\left(U_{1}, m\right)$ is the compressor characteristic. We now have an expression for the pressure $p_{2}$ needed in the model (1). Although (24) may be a somewhat crude approximation for quantitative purposes, the model turns out to capture the essential properties of the compressor, and is in this respect useful in a qualitative analysis. The inlet stagnation temperature $T_{01}$, specific heat capacity $c_{p}$, polytropic efficiency $\eta_{p o l}$ and $\gamma$ are assumed constant. The polytropic efficiency $\eta_{p o l}$ is given by

$$
\eta_{p o l}=\frac{n(\gamma-1)}{\gamma(n-1)}
$$

where $n>\gamma>1$ is the polytropic constant [6]. We assume that $n$, and thus $\eta_{p o l}$, is independent of the pressure ratio. The ideal energy transfer and the losses are shown in figure 2. The curves are calculated for a compressor speed of $N=35.000 \mathrm{rpm}$. The compressor pressure characteristic as calculated from equation (24) is showed in figure 4 .

In figures 2 and 4 the numerical values for the compressor parameters is taken from [7]. Comparing the compressor map in figure 4 with figure 3 in [7], which is based on physical measurements, we see that they are almost similar.

The surge line is the line in the compressor map that divides the map into an area of stable compressor operation and unstable (surge) operation. The line passes through the local maxima of the constant speed lines in the map, and is drawn with a solid line in figure 4 .

\section{Choking}

When the flow reaches the speed of sound at some cross-section of the compression system, the flow chokes. For now it is as-
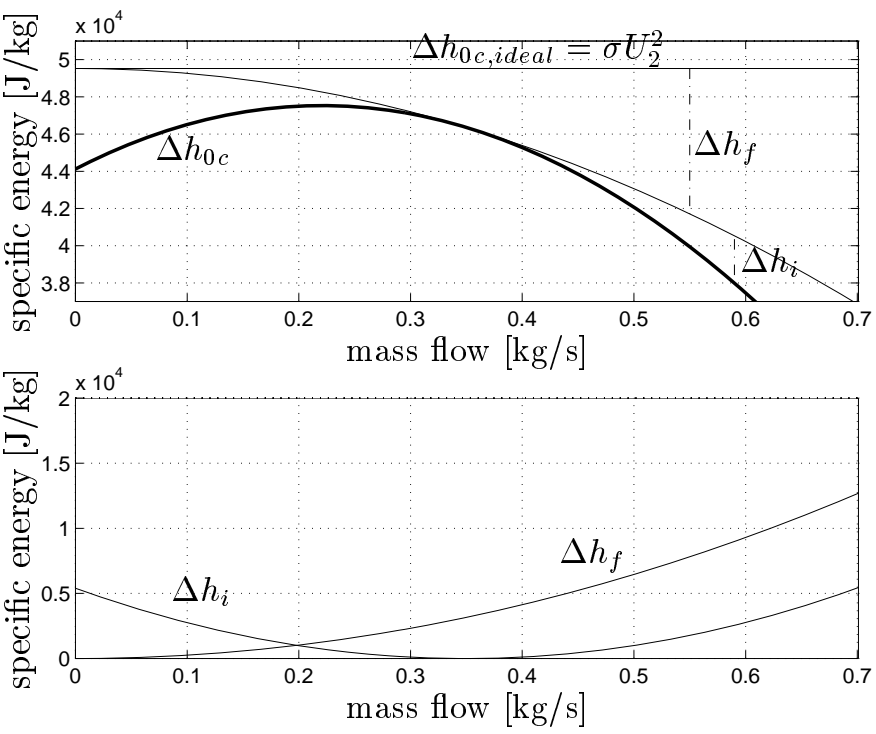

Figure 2: Energy transfer for $N=35.000 \mathrm{rpm}$

sumed that the flow chokes in the impeller. Assuming isentropic flow, [3] calculated the choking flow as

$$
m_{\text {choke }}\left(U_{1}\right)=A_{1} \rho_{01} a_{01}\left[\frac{2+(\gamma-1)\left(\frac{U_{1}}{a_{01}}\right)^{2}}{\gamma+1}\right]^{\frac{(\gamma+1)}{2(\gamma-1)}},
$$

where

$$
\rho_{01}=\frac{p_{01}}{R T_{01}} \text { and } a_{01}=\sqrt{\gamma R T_{01}}
$$

is the inlet stagnation density and inlet stagnation sonic velocity, respectively. It is seen than the choking mass flow is dependent on blade speed $U_{1}$. Thus the impeller can accept a greater limiting mass flow rate at higher rotational speeds. The effect of choking can be seen in figure 4 , where a choke line, also known as a stone wall, has been drawn. In this paper, the effect of choking is treated in a approximate manner. Due to sonic effects, the pressure rise would fall off more gradually when approaching the stone wall than shown in figure 4 .

\section{Dynamic model}

To complete the dynamic model (1), an expression for the throttle mass flow is needed. The mass flow $m_{t}$ through the throttle is modeled as

$$
m_{t}=k_{t} \sqrt{p_{p}-p_{01}}
$$

where $k_{t}$ is the throttle gain proportional to throttle opening and $p_{p}$ is the plenum pressure. The momentum balance of the spool is

$$
J \dot{\omega}=\tau_{t}-\tau_{c}
$$

Using (3) it is seen that

$$
\omega=\frac{2 U_{1}}{D_{1}} \Rightarrow \dot{\omega}=\frac{2 \dot{U}_{1}}{D_{1}}
$$

and thus we get a differential equation for $U_{1}$,

$$
\dot{U}_{1}=\frac{D_{1}}{2 J}\left(\tau_{t}-\tau_{c}\right) \text {. }
$$

The drive torque $\tau_{t}$ may be delivered by a turbine, and will be used as a control variable for speed control. The compressor and spool can only rotate in one direction, and the speed is assumed upper bounded:

$$
0 \leq U_{1}(t)<U_{m}
$$

Using (9) and (31), and inserting equations (24) and (28) in (1), we get the following dynamic model for the compression 
system $^{1}$

$$
\begin{aligned}
\dot{p}_{p} & =\frac{a_{01}^{2}}{V_{p}}\left(m-k_{t} \sqrt{p_{p}-p_{01}}\right) \\
\dot{m} & =\frac{A}{L}\left(\left(1+\frac{\Delta h_{0 c}\left(U_{1}, m\right)}{T_{01} c_{p}}\right)^{\frac{\gamma \eta_{p o l}}{\gamma-1}} p_{01}-p_{p}\right) \\
\dot{U}_{1} & =\frac{D_{1}}{2 J}\left(\tau_{t}-\tau_{c}\right) .
\end{aligned}
$$

\section{Surge control idea}

The reason for equilibria to the left of the surge line being unstable, and causing the compressor to go into surge, is the positive slope of the characteristic in this area. From figure 2 it is seen that the positive slope is due to the incidence losses at low mass flows. From the expression for the incidence loss, equation (11), it is clear that variable blade angle $\beta_{1 b}$ would make it possible to minimize the incidence losses over a range of mass flows. Thus variable inducer blades might be used as a means of surge stabilization.

On the other hand, the maximum energy transfer and minimum incidence loss do not occur for the same mass flow. This is due to the friction losses. The friction shifts the point of maximum energy transfer, and consequently pressure rise, to the left of the point of minimum incidence loss. From this we conclude that the friction losses in fact have a stabilizing effect, and introducing additional fluid friction would move the point of maximum energy transfer to the left. The effect of this, when considering the compressor map in figure 4 is that the surge line will be shifted to the left, and the area of stable compressor operation is expanded.

This motivates us to introduce a valve in series with compressor. The pressure drop over this valve will serve as the control variable, and it will be used to introduce additional friction at low mass flows in order to avoid surge. The use of a close coupled valve for constant speed centrifugal compressor surge control was studied in [4], [12] and [14].

\section{Controller design and stability analysis}

The equivalent compressor characteristic for compressor and close coupled valve is defined as

$$
\Psi_{e}\left(m, U_{1}\right)=\Psi_{c}\left(m, U_{1}\right)-\Psi_{v}(m),
$$

where $\Psi_{v}(m) p_{01}$ is the pressure drop across the CCV and

$$
\Psi_{c}\left(m, U_{1}\right)=\left(1+\frac{\Delta h_{0 c}\left(U_{1}, m\right)}{T_{01} c_{p}}\right)^{\frac{\gamma \eta_{p o l}}{\gamma-1}} .
$$

Assume $p_{0}, m_{0}$ to be the equilibrium values of pressure and mass flow as dictated by the intersection of the throttle and compressor characteristics, and $U_{d}$ to be the desired spool speed. Define the following error variables

$$
\hat{p}=p-p_{0}, \quad \hat{m}=m-m_{0}, \quad \hat{U}=U_{1}-U_{d} .
$$

The equations of motion (33) are now transformed so that the origin becomes the equilibrium under study. Notice that no assumptions are made about the numeric values of $m_{0}$ and $p_{0}$, so that the equilibrium can be on either side of the surge line. By including the CCV (34), the model (33) can be written in the form

$$
\dot{\hat{p}}=\frac{a^{2}}{V_{p}}\left(\hat{m}-\hat{m}_{t}(\hat{p})\right)
$$

\footnotetext{
${ }^{1}$ It is worth noticing that a time varying $U$ is equivalent with a time varying B-parameter [7]. Greitzer's B-parameter as defined in [10] is given by $B=\frac{U_{1}}{2 a_{01}} \sqrt{\frac{V_{p}}{A_{1} L_{c}}}$, where $V_{p}$ is the plenum volume and $L_{c}$ is the length of the compressor and duct. Using (31), a nonlinear differential equation for $B$ can be found.
}

$$
\begin{aligned}
\dot{\hat{m}} & =\frac{A}{L}\left(\left(\hat{\Psi}_{c}(\hat{m}, \hat{U})-\hat{\Psi}_{v}(\hat{m})\right) p_{01}-\hat{p}\right) \\
\dot{\hat{U}}_{1} & =\frac{D_{1}}{2 J}\left(\hat{\tau}_{t}-\hat{\tau}_{c}\right)
\end{aligned}
$$

where a hat denotes transformation to the new coordinates $(36)$, and $(\hat{p} \hat{m} \hat{U})^{\mathrm{T}}=\left(\begin{array}{lll}0 & 0 & 0\end{array}\right)^{\mathrm{T}}$ is the equilibrium.

Theorem 1 The surge control law

$$
\hat{\Psi}_{v}=k_{v} \hat{m}
$$

and the speed control law

$$
\begin{aligned}
\hat{\tau}_{t} & =-k_{p} \hat{U}-k_{i} \hat{I}, \\
\dot{\hat{I}} & =\hat{U},
\end{aligned}
$$

where

$$
k_{p}>0, k_{i}>0 \text { and } k_{v}>\sup _{\hat{U}, \hat{m}}\left\{\frac{\partial \hat{\Psi}_{c}(\hat{m}, \hat{U})}{\partial \hat{m}}\right\}+\delta_{1},
$$

makes the origin of (37) semi-global exponentially stable. That is, desired compressor speed $U_{d}$ is achieved regardless of the equilibrium being to the right or to the left of the original surge line. The design parameter $\delta_{1}>0$ in (40) can be chosen freely. The integral term in (39) is added in order to robustify the controller wrt to unmodeled torques, arising from disc friction, leakages, etc.

Proof 1 Define

$$
\boldsymbol{z} \triangleq\left(\begin{array}{c}
\hat{U} \\
\hat{I}
\end{array}\right) \text { and } \boldsymbol{P} \triangleq\left(\begin{array}{cc}
\frac{2 J}{D_{1}} & \mu \\
\mu & k_{i}
\end{array}\right)
$$

where $\mu>0$ and $k_{i}>0$ are design parameters. Consider the following Lyapunov function candidate

$$
V(\hat{p}, \hat{m}, \hat{U}, \hat{I})=\frac{1}{2}\left(V_{\hat{p}}+V_{\hat{m}}+V_{\text {spool }}\right),
$$

where

$$
V_{\hat{p}}=\frac{V_{p}}{a_{01}^{2} \rho_{01}} \hat{p}^{2}, \quad V_{\hat{m}}=\frac{L}{A_{1} \rho_{01}} \hat{m}^{2} \text { and } V_{\text {spool }}=\boldsymbol{z}^{\mathrm{T}} \boldsymbol{P} \boldsymbol{z} .
$$

As all coefficients in (42) are constant it follows that $V$ is positive definite and radially unbounded, provided that $\mu$ is chosen such that $\boldsymbol{P}>0$, that is

$$
\mu<\sqrt{\frac{2 J k_{i}}{D_{1}}} .
$$

Calculating the time derivative of (42) along the solutions of (37) and accounting for (39) gives

$$
\begin{aligned}
\dot{V}= & \hat{m}\left(\hat{\Psi}_{c}(\hat{m}, \hat{U})-\hat{\Psi}_{v}(\hat{m})\right) \frac{p_{01}}{\rho_{01}}-\frac{1}{\rho_{01}} \hat{p} \hat{m}_{t}(\hat{p})-k_{p} \hat{U}^{2} \\
& +\mu \hat{U}^{2}-\frac{\mu k_{i} D_{1}}{2 J} \hat{I}^{2}-\frac{\mu k_{p} D_{1}}{2 J} \hat{U} \hat{I}-\hat{U} \hat{\tau}_{c}-\frac{\mu D_{1}}{2 J} \hat{I} \hat{\tau}_{c} .(45)
\end{aligned}
$$

The $\hat{U} \hat{\tau}_{c}$-term is the power consumed by the compressor. The last term in (45) can be upper bounded as

$$
-\frac{\mu D_{1}}{2 J} \hat{I} \hat{\tau}_{c} \leq \frac{\mu D_{1}}{2 J}\left(\frac{\sigma D_{2}^{2} U_{m}}{2 D_{1}}\left(\frac{\hat{m}^{2}}{\eta_{1}}+\eta_{1} \hat{I}^{2}\right)+m_{0} \hat{U} \hat{I}\right)
$$

using (9), (32) and Young's inequality. The parameter $\eta_{1}>0$ can be chosen freely. Now, (45) can be upper bounded as

$$
\begin{aligned}
\dot{V} \leq & \hat{m}\left(\hat{\Psi}_{c}(\hat{m}, \hat{U})-\hat{\Psi}_{v}(\hat{m})\right) \frac{p_{01}}{\rho_{01}} \\
& +\frac{\sigma \mu D_{2}^{2} U_{m}}{8 J \eta_{1}} \hat{m}^{2}-\frac{1}{\rho_{01}} \hat{p} \hat{m}_{t}(\hat{p})-\frac{1}{2} \boldsymbol{z}^{\mathrm{T}} \boldsymbol{R} \boldsymbol{z},
\end{aligned}
$$

where

$$
\boldsymbol{R}=\left(\begin{array}{cc}
k_{p}-\mu & \frac{\mu}{4 J}\left(D_{1} k_{p}-\frac{\sigma D_{2}^{2} m_{0}}{4}\right) \\
\frac{\mu}{4 J}\left(D_{1} k_{p}-\frac{\sigma D_{2}^{2} m_{0}}{4}\right) & \frac{\mu}{2 J}\left(k_{i} D_{1}-\frac{\sigma D_{2}^{2} U_{m} \eta_{1}}{4}\right)
\end{array}\right)
$$


Demanding $\boldsymbol{R}>0$ gives the following conditions on $\eta_{1}$ and $\mu$

$$
\begin{aligned}
& \eta_{1}<\frac{4 k_{i} D_{1}}{\sigma D_{2} U_{m}} \text { and } \\
& \mu<\min \left\{k_{p}, \frac{8 J k_{p} k_{i} D_{1}-2 J k_{p} \sigma D_{2} U_{m} \eta_{1}}{8 J k_{i} D_{1}-2 J \sigma D_{2} U_{m} \eta_{1}+\left(D_{1} k_{p}-\frac{\sigma D_{2}^{2} m_{0}}{4}\right)^{2}}\right\}
\end{aligned}
$$

It is assumed that $\hat{m}_{t}$ satisfies the sector condition

$$
\hat{p} \hat{m}_{t}(\hat{p})>\delta_{2} \hat{p}^{2}
$$

that is, the throttle is assumed passive as in [15]. As $\hat{p} \hat{m}_{t}(\hat{p})$ is of order $\frac{3}{2}$ in $\hat{p},(51)$ does not hold globally. However for a given $\hat{p}_{\max }$ such that

$$
|\hat{p}(t)| \leq p_{m} \forall t>0
$$

it will always be possible to chose $\delta_{2}$ small enough for (51) to hold for $|\hat{p}(t)| \leq \hat{p}_{\max }$. Now, the CCV pressure drop $\Psi_{v}(\hat{m})$ is to be chosen such that for the first term in (47), the condition

$$
-\hat{m}\left(\hat{\Psi}_{c}(\hat{m}, \hat{U})-\hat{\Psi}_{v}(\hat{m})\right) \frac{p_{01}}{\rho_{01}}>0 \forall \hat{U}
$$

is satisfied. Since $\frac{p_{01}}{\rho_{01}}>0$, sufficient conditions for (53) to hold is

$$
\begin{aligned}
-\left.\left(\hat{\Psi}_{c}(\hat{m}, \hat{U})-\hat{\Psi}_{v}(\hat{m})\right)\right|_{\hat{m}=0} & =0 \text { and } \\
\frac{\partial}{\partial \hat{m}}\left(-\hat{\Psi}_{c}(\hat{m}, \hat{U})+\hat{\Psi}_{v}(\hat{m})\right) & >0 .
\end{aligned}
$$

It can be recognized that

$$
\begin{aligned}
\hat{\Psi}_{c}(0, \hat{U}) & =\Psi_{c}\left(m_{0}, U_{d}\right)-\Psi_{c}\left(m_{0}, U_{d}\right)=0, \\
\hat{\Psi}_{v}(\hat{m}) & =k_{v} \hat{m} \Rightarrow \hat{\Psi}_{v}(0)=0,
\end{aligned}
$$

and thus (54) is satisfied. From (55), we get

$$
-\frac{\partial}{\partial \hat{m}} \hat{\Psi}_{c}(\hat{m}, \hat{U})+k_{v}>0
$$

and it follows that choosing $k_{v}$ according to

$$
k_{v}>\sup _{\hat{U}, \hat{m}}\left\{\frac{\partial \hat{\Psi}_{c}(\hat{m}, \hat{U})}{\partial \hat{m}}\right\},
$$

guarantees that (55), and thereby (53) being satisfied. Moreover, if chose

$$
k_{v}>\sup _{\hat{U}, \hat{m}}\left\{\frac{\partial \hat{\Psi}_{c}(\hat{m}, \hat{U})}{\partial \hat{m}}\right\}+\delta_{1}
$$

where $\delta_{1}>0$, we get $\hat{m} \Psi_{v}(\hat{m})>\delta_{1} \hat{m}^{2}$, and (53) is modified to

$$
-\hat{m}\left(\hat{\Psi}_{c}(\hat{m}, \hat{U})-\hat{\Psi}_{v}(\hat{m})\right) \frac{p_{01}}{\rho_{01}}>\frac{p_{01}}{\rho_{01}} \delta_{1} \hat{m}^{2} \forall \hat{U} .
$$

Consequently $\dot{V}$ can now be upper bounded as

$$
\dot{V} \leq-\left(\frac{p_{01}}{\rho_{01}} \delta_{1}-\frac{\sigma \mu D_{2}^{2} U_{m}}{8 J \eta_{1}}\right) \hat{m}^{2}-\delta_{2} \hat{p}^{2}-\frac{1}{2} \boldsymbol{z}^{\mathrm{T}} \boldsymbol{R} \boldsymbol{z} \quad \forall \hat{m}, \hat{p}, \boldsymbol{z}
$$

We now set out to compare the coefficients of $V$ and $\dot{V}$. The cross terms in $\hat{U}$ and $\hat{I}$ are upper bounded using Young's inequality,

$$
\begin{aligned}
\mu \hat{U} \hat{I} & \leq \frac{\mu}{2}\left(\frac{\hat{U}^{2}}{\eta_{2}}+\eta_{2} \hat{I}^{2}\right) \\
-\frac{\mu\left(4 D_{1} k_{p}-\sigma D_{2}^{2} m_{0}\right)}{8 J} \hat{U} \hat{I} & \leq \frac{\mu\left(4 D_{1} k_{p}-\sigma D_{2}^{2} m_{0}\right)}{16 J} \times \\
& \left(\hat{U}^{2} / \eta_{3}+\eta_{3} \hat{I}^{2}\right)
\end{aligned}
$$

where $\eta_{2}>0$ and $\eta_{3}>0$ are constants that can be chosen freely. Using (63) and (64) and comparing the coefficients in (42) and (62), it can be recognized that if the following inequalities are satisfied for some $\alpha>0$

$$
\delta_{1}-\frac{\sigma \mu D_{2}^{2} U_{m} \rho_{01}}{8 J \eta_{1} p_{01}}>\alpha \frac{L}{A \rho_{01}} \quad, \quad \delta_{2}>\alpha \frac{V_{p}}{a_{01}^{2} \rho_{01}}
$$

$$
\begin{aligned}
\frac{k_{p}}{2 D_{1}}-\frac{\mu}{2}-\frac{\mu\left(4 D_{1} k_{p}-\sigma D_{2}^{2} m_{0}\right)}{16 J \eta_{3}} & >\alpha\left(\frac{J}{D_{1}^{2}}+\frac{\mu}{2 \eta_{2}}\right) \\
\frac{\mu k_{i}}{2}-\frac{\mu\left(4 D_{1} k_{p}-\sigma D_{2}^{2} m_{0}\right)}{16 J} \eta_{3} & >\alpha\left(\frac{k_{i}}{D_{1}}+\frac{\alpha \eta_{2}}{2}\right)
\end{aligned}
$$

the following holds

$$
\dot{V} \leq \alpha V \Rightarrow V(t) \leq V(0) e^{-\alpha t} .
$$

If $\eta_{1}$ is chosen according to (49), and $\delta_{1}$ is chosen according to $\delta_{1}>\frac{\sigma \mu D_{2}^{2} U_{m} \rho_{01}}{8 J \eta_{1} p_{01}}$, and $k_{v}$ is chosen so that $k_{v}>$ $\sup _{\hat{U}, \hat{m}}\left\{\frac{\partial \hat{\Psi}_{c}(\hat{m}, \hat{U})}{\partial \hat{m}}\right\}+\delta_{1}$, and $\mu$ is chosen according to (44) and (50), then the inequalities (65)-(67) are satisfied for some $\alpha>0$. By (68) the origin of (37) is exponentially stable. Due to assumption (52), the stability result holds whenever $|\hat{p}(0)| \leq p_{m}$, and thus the origin is semi-global exponential stable.

Notice that the parameter $\alpha$ can be used to calculate a lower bound on the convergence rate of the system.

\section{Simulations}

In order to model the compressor pressure rise for negative mass flow (deep surge) the following assumption is made:

$$
\Delta h_{0 c}\left(U_{1}, m\right)=\Delta h_{0 c}\left(U_{1},-m\right),
$$

the energy transfer is symmetric in $m$. This assumption is not based on any physical considerations, but the resulting characteristic bear resemblance to real compressor characteristic, e.g. the one in [7]. In figure 3 the response of the system during surge is showed. The set point for compressor speed was $U_{1 d}=150 \mathrm{~m} / \mathrm{s} \Rightarrow N \approx 50.000 \mathrm{rpm}$ and the speed control law (39) parameters were set to $k_{p}=k_{i}=0.07$. The throttle gain was set to $k_{t}=0.0075$ which gives an unstable equilibrium to the left of the surge line. This simulation is also showed in figure 4 , where the pressure rise has been plotted versus the mass flow in the compressor characteristic. As can be seen, the compressor undergoes severe (deep) surge oscillations, and the compressor speed oscillates around the desired value. These variations in spool speed was first described in [5].
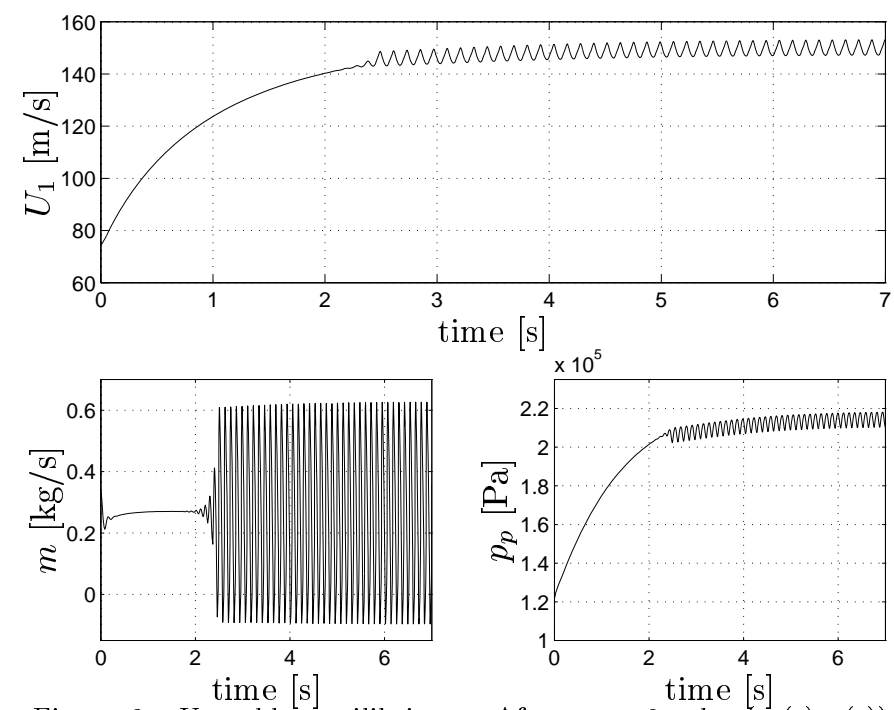

Figure 3: Unstable equilibrium. After $t \approx 2 \mathrm{~s}$ the $(m(t), p(t))$ trajectory crosses the surge line, resulting in deep surge.

Now, the surge controller (38) is used with $k_{v}=0.2$. The speed set point, speed controller parameters and throttle gain are as before. The results are shown in figure 5. The desired speed is reached and the surge oscillations are eliminated. As previously mentioned there is a loss associated with the CCV 


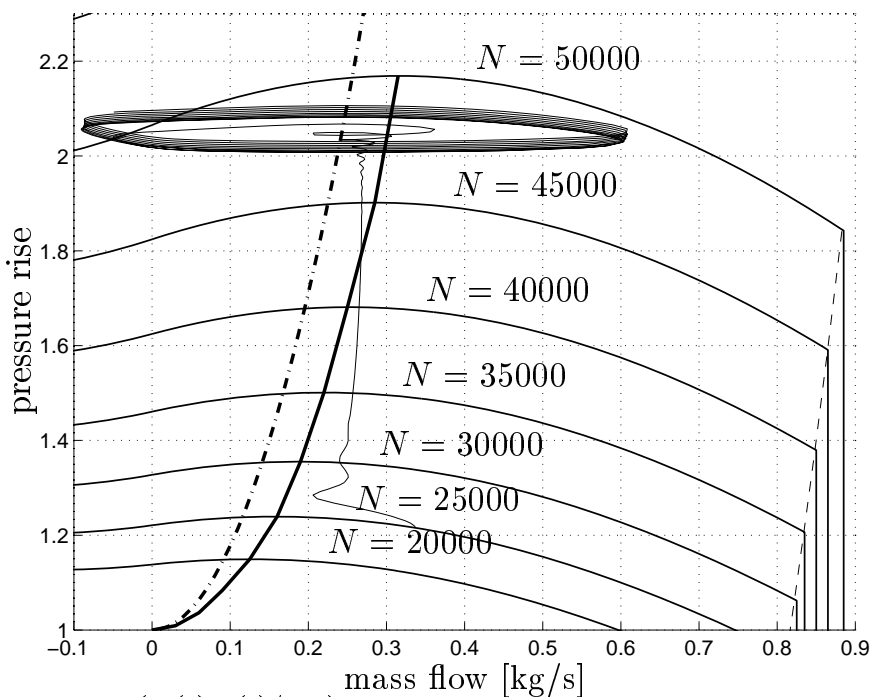

Figure 4: $\left(m(t), p(t) / p_{01}\right)$-trajectory plotted in the compressor characteristic. The throttle line is plotted with a dash-dot line, the surge line with a solid line and the stonewall with a dashed line. $N$ is the compressor speed in rpm. The surge cycle is clearly visible.

control approach. The pressure drop over the valve is showed in the upper right corner of figure 5 . At equilibrium the pressure drop for this particular case is at ca. $5 \mathrm{kPa}$. Compared to the pressure rise over the compressor at this equilibrium, well over $200 \mathrm{kPa}$ this seems little when taken into account that the compressor now is operating in an area of the compressor map previously not possible. The CCV loss is dependent on the controller gain $k_{v}$. In this simulation the gain was set to $k_{v}=0.2$ to dominate the maximum positive slope of the compressor characteristic.
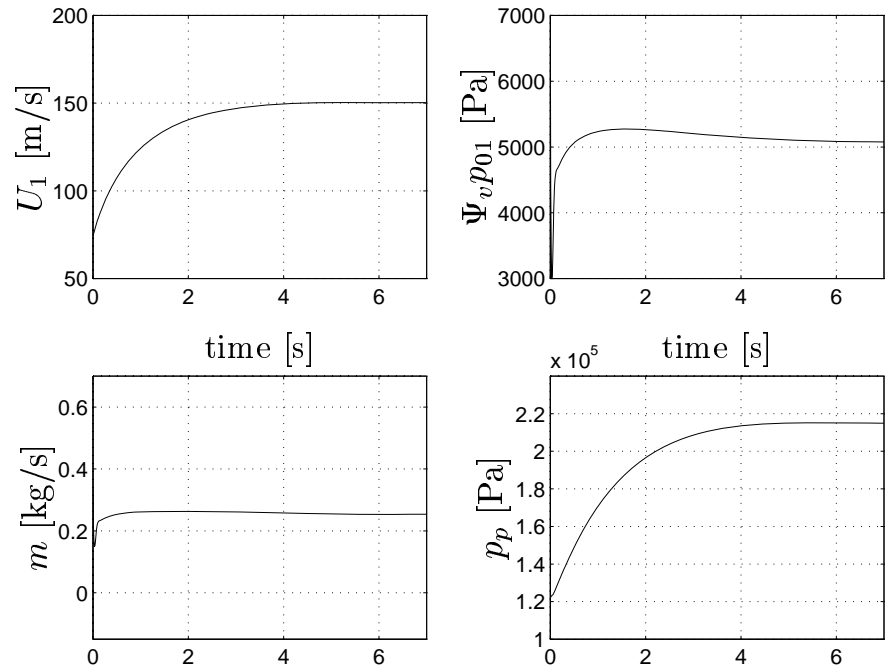

Figure 5: time $[\mathrm{S}$. $]$ situation as in figure 3 , with time surge $[\mathrm{S}]$ ntrol

\section{Conclusion}

A surge control law and a PI speed control law for a centrifugal compression system have been developed. The modeling of the compressor characteristic was based on energy losses in the compressor stage. A close coupled valve was chosen as an actuator for the surge control. Using Lyapunovs method, the systems equilibrium was showed to be semi-global exponentially stable. Through simulations it was confirmed that the compressor can operate stable and reach desired speed in the previous unstable area to the right of the surge line in the compressor map.

\section{References}

[1] O.E. Baljé. A contribution to the problem of designing radial turbomachines. Transactions of the ASME, 74:451$472,1952$.

[2] B. de Jager. Rotating stall and surge control : A survey. In Proceedings of the 35th Conference on Decision and Control, pages 1857-1862, New Orleans, LA, 1995.

[3] S.L. Dixon. Thermodynamics of turbomachinery. Pergamon Press, 3rd edition, 1978.

[4] J.L. Dussourd, G.W. Pfannebecker, and S.K. Singhania. An experimental investigation of the control of surge in radial compressors using close coupled resistances. Journal of Fluids Engineering, 99:64-76, 1977.

[5] K.M. Eveker and C.N. Nett. Model development for active surge control rotating stall avoidance in aircraft gas turbine engines. In Proceedings of the 1991 American Control Conference, pages 3166-3172, 1991.

[6] T.B. Ferguson. The centrifugal compressor stage. Butterworths, London, 1963.

[7] D.A. Fink, N.A. Cumpsty, and E.M. Greitzer. Surge dynamics in a free-spool centrifugal compressor system. Journal of Turbomachinery, 114:321-332, 1992.

[8] J.T. Gravdahl and O. Egeland. Compressor surge control using a close-coupled valve and backstepping. In Proceedings of the 1997 American Control Conference, Albuquerque, NM, June 1997.

[9] J.T. Gravdahl and O. Egeland. Passivity based compressor surge control using a close-coupled valve. In A. Isidori and F. Allgöwer, editors, Proceedings of the $199^{\text {r }}$ COSY Workshop on Control of Nonlinear and Uncertain Systems, pages 139-143, Zurich, Switzerland, January 1997.

[10] E.M. Greitzer. Surge and Rotating stall in axial flow compressors, Part I: Theoretical compression system model. Journal of Engineering for Power, 98:190-198, 1976.

[11] E.M. Greitzer. The stability of pumping systems - The 1980 Freeman scolar lecture. Journal of Fluids Engineering, 103:193-242, 1981.

[12] W.M. Jungovski, M.H. Weiss, and G.R. Price. Pressure oscillations occuring in a centrifugal compressor system with and without passive and active surge control. Journal of Turbomachinery, 118:29-40, 1996.

[13] K.E. Hansen, P. Jørgensen, and P.S. Larsen. Experimental and theoretical study of surge in a small centrifugal compressor. Journal of Fluids Engineering, 103:391-394, 1981.

[14] J.E. Pinsley, G.R. Guenette, A.H. Epstein, and E.M. Greitzer. Active stabilization of centrifugal compressor surge. Journal of Turbomachinery, 113:723-732, 1991.

[15] J.S. Simon and L. Valavani. A Lyapunov based nonlinear control scheme for stabilizing a basic compression system using a close-coupled control valve. In Proceedings of the 1991 American Control Conference, pages 2398-2406, 1991.

[16] J.S. Simon, L. Valavani, A.H. Epstein, and E.M. Greitzer. Evaluation of approaches to active compressor surge stabilization. Journal of Turbomachinery, 115:57-67, 1993.

[17] N. Watson and M.S. Janota. Turbocharging the internal combustion engine. MacMillan, 1982.

[18] F.M. White. Fluid mechanics. McGraw-Hill, New York, 2nd edition, 1986. 